HEP/123-qed

\title{
A new volume-of-fluid formulation for surfactants and simulations of drop deformation under shear at a low viscosity ratio
}

\author{
Yuriko Y. Renardy and Michael Renardy \\ Department of Mathematics and ICAM \\ 460 McBryde Hall, Virginia Tech \\ Blacksburg, VA 24061-0123, USA \\ Vittorio Cristini \\ Department of Chemical Engineering and Materials Science \\ 151 Amundson Hall, 421 Washington Avenue S.E. \\ University of Minnesota \\ Minneapolis, MN 55455-0132 USA
}

(May 10, 2001)

(Submitted to European J. Mech B/Fluids)

\begin{abstract}
A numerical algorithm for the linear equation of state is developed for the volume-of-fluid interface-tracking code SURFER++, using the continuous surface stress formulation for the description of interfacial tension. This is applied to deformation under simple shear for a liquid drop in a much more viscous matrix liquid. We choose a Reynolds number and capillary number at which the drop settles to an ellipsoidal steady state, when there is no surfactant. The viscosity ratio is selected in a range where experiments have
\end{abstract}


shown tip streaming when surfactants are added. Our calculations show that surfactant is advected by the flow and moves to the tips of the drop. There is a threshhold surfactant level, above which the drop develops pointed tips, which are due to surfactant accumulating at the ends of the drop. Fragments emitted from these tips are on the scale of the mesh size, pointing to a shortcoming of the linear equation of state, namely that it does not provide a lower bound on interfacial tension. One outcome is the possibility of an unphysical negative surface tension on the emitted drops.

47.55.Dz, 47.11.+j, 47.20.Gv

Typeset using REVTEX 


\section{INTRODUCTION}

When a drop of viscous liquid is placed in a matrix liquid of equal viscosity and sheared, the subsequent motion leads to ellipsoidal shapes for steady-state solutions, and dumbbell shapes for unsteady evolution to breakup. On the other hand, when the viscosity of the drop is much less than the matrix liquid, then it is known [1-3] that steady-state solutions produce strongly distorted interfaces with high curvature at the ends. In this paper, we investigate the first effect of surfactants on drop evolution and breakup for the drop to matrix viscosity ratio of 0.05 . This is chosen as a representative value where the drop deforms to a typical almond-shape with high curvature at the ends and tip-streaming is observed in experiments when surfactant is present, see [4]. In this paper, we cover three issues. The first is the investigation of a novel algorithm for the volume-of-fluid scheme for the incorporation of surfactants. Secondly, we show that when the amount of surfactant is increased, there is a critical value above which the drop develops tips and droplets emerge. Thirdly, when the amount of surfactant reaches a high level, a normal breakup mode occurs in place of droplet production from tips.

In Section II, the basic steps in our numerical method are summarized. The key step is the calculation of the interfacial tension force, which is described in detail in Section III. The performances of the continuous surface stress formulation and the continuous surface force formulation are compared. The role of mollifications to the color function, which have been used in the past, is discussed in relation to the sensitivity of the numerical simulations of drop shapes with high curvature. We develop our algorithm for the linear dependence of interfacial tension on surfactant concentration in section IV. The key difficulty in formulating any algorithm for surfactants within the framework of the volume-of-fluid scheme is that the fluid properties are expressed most naturally and easily in terms of volumes rather than surfaces. As our first step in the investigation of the effect of surfactants, the linear equation of state is the simplest to implement. In Section V, the effect of reduction in interfacial tension is investigated. An example is given for the formation of tipped ends 
which emit small drops. However, the linear equation of state allows the interfacial tension to decrease to negative values. Future work will address the manner in which nonlinear

equations of state impose the concept of maximal packing $[5,6]$ and prevent the unphysical decrease in interfacial tension that we observe here.

In all of our computations, we use a computational box of dimensions $3 \times 0.5 \times 1$. Periodic boundary conditions are imposed in the $x$ and $y$ directions, and the top and bottom plates move with uniform speed \pm 0.5 . The drop is initially centered in the box with radius $a=0.125$. The drop and matrix fluid have equal density and viscosity ratio 0.05 . The dimensionless parameters are the Reynolds number

$$
R e=\frac{\rho \dot{\gamma} a^{2}}{\eta}
$$

where $\dot{\gamma}$ is the shear rate (normalized to 1 in the computations), and $\eta$ is the viscosity of the matrix fluid, and the capillary number

$$
C a=\frac{\eta \dot{\gamma} a}{\sigma}
$$

If there is surfactant present, and surface tension is variable, we define $C a$ to be the capillary number based on the value $\sigma$ of the surface tension coefficient without surfactant.

\section{SURFER++}

Our code SURFER++ is composed of three parts: a volume-of-fluid VOF method to track the interface $[7,8]$ based on piecewise linear interface reconstruction, a projection method to solve the Navier-Stokes equations on the MAC grid, and a continuum method for modeling the interfacial tension. The novel aspects in this paper concern the VOF component and the modeling of interfacial tension; both of these are discussed in detail in subsequent sections. The details of the original code SURFER are given in [9], the interface reconstruction scheme is discussed in [10], and new capabilities of SURFER ++ to handle low Reynolds number flows are described in [11-13]. 
The density $\rho$ and the viscosity $\mu$ of each fluid is a constant in each fluid. A concentration (or color) function $C$ is used to track the interface:

$$
C(\mathbf{x})=\left\{\begin{array}{l}
1 \text { fluid } 1 \\
0 \text { fluid } 2 .
\end{array}\right.
$$

This concentration function is transported by the velocity field $\mathbf{u}$. The fluids are incompressible: $\nabla \cdot \mathbf{u}=0$, and governed by the Navier-Stokes equation:

$$
\rho\left(\frac{\partial \mathbf{u}}{\partial t}+\mathbf{u} \cdot \nabla \mathbf{u}\right)=-\nabla p+\nabla \cdot \mu \mathbf{S}+\mathbf{F}
$$

where $\mathbf{S}$ is the viscous stress tensor. The body force $\mathbf{F}$ includes the interfacial tension force.

The temporal discretization is based on Chorin's projection method, which decouples the pressure equation. A semi-implicit Stokes solver has been incorporated. The spatial discretization is a Cartesian mesh of rectangular cells. It is a finite difference mesh known as the MAC grid. The density and viscosity for each cell are given by $\rho=C \rho_{1}+(1-C) \rho_{2}$ and $\mu=C \mu_{1}+(1-C) \mu_{2}$, where subscripts refer to fluids 1 and 2 .

A piecewise linear interface calculation (PLIC) method is used to reconstruct the interface position. The approximate normal $\mathbf{n}$ to the interface in each cell is equal to the discrete gradient of the volume fraction field: $\mathbf{n}=\nabla C /|\nabla C|$. The final step of the VoF method is to evolve the volume fraction field $C$. At the nth timestep, the interface is reconstructed, the velocity at the interface is interpolated linearly and then the new interface position for the $(\mathrm{n}+1)$ th timestep is calculated via a Lagrangian method: $\mathbf{x}^{n+1}=\mathbf{x}^{n}+\mathbf{u}(\Delta t)$.

The entire code has been parallelized; data on the scalability and timings are given in $[14]$.

\section{ALGORITHMS FOR INTERFACIAL TENSION AS A BODY FORCE}

In the VOF method, interfacial tension is posed as a body force over the discretized cells which contain the interface. Thus, in the Navier-Stokes equation (2.1), the body force $\mathbf{F}$ includes the interfacial tension force $\mathbf{F}_{\mathbf{s}}=\sigma \kappa \mathbf{n}_{\mathbf{s}} \delta_{s}$, where $\sigma$ is the interfacial tension, $\kappa$ 
is the mean curvature and $\mathbf{n}_{\mathbf{s}}$ is the normal to the interface. Two such formulations have been implemented in SURFER. The first is the continuous surface force (CSF) formulation [15], in which $\mathbf{f}_{s}=\sigma \kappa \mathbf{n}_{S}$, and $\mathbf{F}_{s}=\mathbf{f}_{s}|\nabla C|$, where $\sigma$ is the interfacial tension, $\kappa$ is the mean curvature and $\mathbf{n}_{S}$ is the normal to the interface. The second is the continuous surface stress (CSS) formulation [9], in which

$$
\mathbf{F}_{s}=\nabla \cdot \mathbf{T}=\sigma \delta_{\mathbf{S}} \kappa \mathbf{n}_{\mathbf{S}} \quad \text { and } \quad \mathbf{T}=\left[\left(\mathbf{1}-\mathbf{n}_{\mathbf{S}} \otimes \mathbf{n}_{\mathbf{S}}\right) \sigma \delta_{\mathbf{S}}\right] .
$$

Both methods are equivalent at the continuum level.

The concentration function is discontinuous across the interface, but in SURFER, this is replaced by a smoothed color function $\tilde{C}(\mathbf{x})$ which varies from 0 to 1 over a distance $\mathcal{O}(\epsilon)$, where $\epsilon$ is of the order of the mesh. In SURFER, $\epsilon$ is chosen to be twice the mesh size. The mollified color function $\tilde{C}$ is obtained by convolving $\mathrm{C}$ with a kernel $\mathbf{K}(\mathbf{x}, \epsilon)$ :

$$
\tilde{C}(\mathbf{x})=\int_{\Omega} C\left(\mathbf{x}^{\prime}\right) \mathbf{K}\left(\mathbf{x}^{\prime}-\mathbf{x}, \epsilon\right) d \mathbf{x}^{\prime}
$$

SURFER uses the kernel function $\mathbf{K}_{8}$ introduced in [16] and calculates the mollified color function by carrying out two smoothing steps.

This smoothing was introduced to suppress spurious currents which arise from inaccurate discrete approximations to the interface normal and curvature. These spurious currents do not disappear with mesh refinement. Converged results can be achieved if smoothing is carried out over a distance which is large relative to the mesh size [17]. This is not realistic in practical simulations. In our simulations, the spurious currents are insignificant relative to the true velocities, in contrast to static situations where the true velocities are zero. The smoothing can, however, affect the solution in regions of high curvature, such as prolonged jets or tips, where smoothing out interfacial tension hurts the simulation.

The effect of smoothing is illustrated in figure 2, where $R e=1, C a=0.625, \lambda=0.05$. The computational domain is $3 \times 0.5 \times 1$, timesteps $\Delta t=10^{-3}$, mesh $192 \times 32 \times 64$. The results are identical with timesteps $0.2 \times 10^{-3}$. The mother drop radius is $a=0.125$. Drop deformations are shown for $t=5,10,15 \mathrm{~s}$. The results were obtained with smoothing for both 
CSS and CSF and without smoothing for CSS. In the case of no smoothing, it is essential to use the conservation form of the surface tension force in the CSS method. CSF without smoothing produces unphysical surface forces and unacceptable results, and therefore, CSF is not pursued in the development of the surfactant algorithm in section IV. Our results show that, as time progresses, smoothing introduces artificial tips at the ends of the drop, which eventually spawn droplets. The smoothing diminishes surface tension in regions of high curvature, which mimics the effect of surfactants accumulating at the ends of the drop and induces tipstreaming as a numerical artifact. When smoothing is removed, this artifact disappears, as evident in the figure.

\section{ALGORITHM FOR LINEAR DEPENDENCE OF INTERFACIAL TENSION ON SURFACTANT CONCENTRATION}

We use the Continuous Surface Stress formulation (cf. equation (3.1)). The CSS formulation for the original code SURFER, in which $\sigma$ is a constant, remains basically the same for the case of non-constant $\sigma$. We need to modify the surface stress $\mathbf{T}$ to replace $\sigma$ with $\sigma-E \Gamma$, where the elasticity number $E$ is a measure of the sensitivity of the surface tension to the surfactant concentration $\Gamma[5]$.

In the volume-of-fluid scheme, properties of the fluid are identified with volumes rather than surfaces. As part of the initial condition, we define two additional spheres, one interior to the drop with radius $h / 2$ smaller, and one exterior to the drop with radius $h / 2$ larger, where $h$ is sufficiently small. Figure 3 illustrates the initial condition. The outer sphere is defined by a VOF function $c_{+}$at the continuous level, which is 0 outside the sphere and 1 inside the sphere. Similarly, the inner sphere is defined by $c_{-}$, which is 0 outside it and 1 inside it. The surfactant lives between the outer and inner spheres, and surfactant concentration $\Gamma$ is a constant multiple of $c_{+}-c_{-}$, which is 1 in the spherical annulus between the outer and inner spheres, and 0 outside. The three interfaces will be tracked during the numerical simulation. 
The discrete version of the term $\sigma \delta_{s}$ in the continuous surface stress $\mathbf{T}$ is to be replaced by $\sigma|\nabla C|-K\left(c_{+}-c_{-}\right)$, where $\delta_{s}$ is $|\nabla C|$, which integrates to 1 across the interfacial region. Upon integration from the inner to the outer sphere along the radial direction, this expression becomes $\sigma-K h$. Therefore, $K h=E \Gamma$. Therefore, we arrive at

$$
\mathbf{T}=\left(\mathbf{1}-\mathbf{n}_{S} \otimes \mathbf{n}_{S}\right)\left[\sigma|\nabla C|-\frac{E \Gamma}{h}\left(c_{+}-c_{-}\right)\right]
$$

We denote the reduction factor by

$$
r=\frac{E \Gamma}{\sigma}=1-\frac{\text { surface tension with surfactant }}{\text { surface tension without surfactant }}
$$

This reduction factor is the quantity which is actually used by the code; $r$ and $h$ are input parameters, and we then have $K=\sigma r / h$.

The algorithm is tested for a variety of surfactant layer depths $h$. Figure 4 shows the evolution of the Taylor deformation parameter

$$
D f=(L-B) /(L+B)
$$

calculated from the side-view length $L$ and breadth $B$ at $R e=10, C a=0.16, r=0.2$. The deformations correspond to the displayed velocity fields. Thus, the surfactant layer depth can be chosen to equal the smallest mesh cell length, in cases like this where drop deformation does not involve prolonged simulation of high curvatures.

When the drop evolves to a tipped shape, however, the choice of the surfactant layer thickness becomes important. If it is chosen too large, the simulation can "lose" the surfactant because it migrates off the drop by moving to neighboring cells that are not taken into account when stresses due to surfactants are computed. This can be thought of as a numerically induced desorption, in which the surfactant moves off the interfacial cells as high curvature regions evolve. An example of the effect of the initial surfactant layer is shown in Figure 5. In the first sequence of pictures $(h=\Delta x / 3)$, some of the surfactant has numerically migrated from the drop (without taking any fluid with it) before the final frame, and hence the separation of small droplets of liquid (which is present with $h=\Delta x / 10$ ) is not 
observed. Our results in subsequent sections are obtained with sufficiently thin initial surfactant layers, and we shall show the location of the surfactants with each result to convince the reader that there is no numerical migration.

In the regime where the drop evolves to a tipped shape, very high concentrations of surfactant occur at the tips. The linear equation of state allows surface tension to become negative, and this leads to a mathematically ill-posed problem. Numerically, this manifests itself in high sensitivity of the results to the accuracy of the pressure iteration. In all our runs reported in the next section, we set the thickness of the initial surfactant layer equal to 0.0001 (roughly two orders of magnitude less than the mesh size), and we set the tolerance bound for the pressure iteration in SURFER equal to $10^{-12}\left(10^{-6}\right.$ was sufficient for our earlier simulations).

\section{EFFECT OF REDUCTION FACTOR}

We begin with the case of zero surfactants at $R e=1, C a=0.2, \lambda=0.05$. As the amount of surfactant is increased, figure 6 shows that tipstreaming begins at some critical value. As the amount of surfactant increases, the drop becomes even more elongated. Experiments of [4] show very elongated drops with little droplets streaming off the ends when the amount of surfactant exceeds a certain minimum. This is illustrated in our simulations for reduction factor 0.3 (see Figures 7,8). Tipstreaming ceases at much higher levels of the surfactant, where the drop may become sufficiently elongated to undergo a "normal" breakup. This is shown for our simulations at reduction factor 0.5 (Figures 9, 10). It has been suggested [18] that at high levels of surfactant concentration, the drop is uniformly covered and this inhibits tip streaming. The emitted drops are expected to have radii much smaller than that of the mother drop: in [4], the mother drops are on the order of millimeters, and the emitted drops on the order of 10 microns. This places the droplets beyond the resolution which we are able to attain at this time.

The results for $r=0.3$ are shown in Figure 7 for meshes of $128 \times 32 \times 64$ and $192 \times 48 \times$ 
96. The drop develops elongated tips which eventually emit fragments. With every mesh refinement, the details of what happens at the tip are at the level of the cell size, indicating that the linear equation of state does not contain enough physics to determine the details of the emitted drops. We conclude this from the following three observations. First, the time when the fragments are emitted depends on the mesh. Secondly, the size of the droplets is on the scale of the mesh. For instance, the small droplets visible in the last subfigure of Figure 7 have volume of approximately 0.89 times a mesh cell. Thirdly, fragments carry a sizable amount of surfactant and have negative surface tension. The fragments in the last subfigure each carry approximately $6 \%$ of the total amount of surfactant and have an "effective surface tension" (calculated by presuming them to be spherical) of minus 5 times the surface tension without surfactant. On the other hand, the results confirm some of the features observed in tipstreaming. The drops form elongated ends, and surfactant accumulates at these ends. This is further illustrated by Figure 8, which shows the distribution of the amount of surfactant in a vertical cross-section through the middle of the drop.

Figures 9 and 10 show the results for the higher reduction factor $r=0.5$, at which the effective capillary number is high enough to induce normal breakup mode. On the more refined mesh, we observe that tips first form at the ends of the elongated drop, but the ends lose their points once the normal breakup sequence is entered. The flow structure is no longer such that it would pull the tips out further. As this happens, the ends develop bulbous shapes, and indications of tipstreaming disappear.

\section{SCALINGS FOR EMITTED DROPS}

Our computations based on the linear equation of state, and at the resolution we presently have, fall short of predicting the properties of droplets emanating from the tips. We believe that this is due to the fact that surface tension is permitted to become negative in the simulations, thus removing the mechanism by which droplets are pinched off. In reality, there is a small but positive value of surface tension even at a high surfactant concentration. 
The incorporation of additional physics for this in numerical simulations is left as an open problem. We now derive the scalings which such an investigation should retrieve.

Assume that a number $N$ of monodisperse fragments of radius $D$ are produced by tipstreaming. The capillary number of a fragment is

$$
C a_{D}=\frac{\mu \dot{\gamma} D}{\sigma_{D}}
$$

where $\sigma_{D}$ is surface tension on a fragment. Imposing that the capillary number of a fragment is critical, we get a first condition

$$
C a_{c}=C a \frac{D}{a} \frac{\sigma}{\sigma_{D}}
$$

after rescaling, where $C a=\mu \dot{\gamma} a / \sigma$. Here, $C a_{c}$ is the critical capillary number for onset of drop breakup.

A second condition comes from conservation of surfactant. Assume that tipstreaming ends when the mother drop has been fully depleted of surfactant. Then

$$
N \frac{\Gamma_{D}}{\Gamma}=\left(\frac{D}{a}\right)^{-2}
$$

This yields four unknowns $N, D / a, \Gamma_{D} / \Gamma$, and $\sigma_{D} / \sigma$. We have two conditions (6.2)(6.3) relating these unknowns. In addition, we expect that there is an upper bound on surfactant concentration when the surfactant "completely covers" the surface area; this yields a value for $\Gamma_{D} / \Gamma$. The value of surface tension at this limiting surfactant concentration determines $\sigma_{D} / \sigma$. The verification of these scalings would provide conclusive understanding of tipstreaming.

\section{CONCLUSIONS}

We have developed a novel algorithm for the simplest equation of state for surfactants, within the volume-of-fluid interface tracking method. We have investigated in detail the formulation for the interfacial tension force as a body force, and in particular, the mollification of the concentration function is examined in the context of drops with tipped ends. It is 
found that mollification reduces interfacial tension noticeably in regions of high curvature, which introduces tip-streaming as a numerical artifact. We have chosen therefore to use the continuous surface stress formulation with no mollification.

The evolution at Reynolds number 1 and a drop-to-matrix viscosity ratio 0.05 is examined. It is found that the surfactant is advected by the flow to the ends of the drop. At some critical value of the reduction factor, the ends of the drop develop tips. The tips emit fragments on the scale of the mesh size. We believe that the actual size of drops emitted in tip streaming is determined by the amount of surface tension which remains when the surfactant reaches its maximum concentration. In the model used in our simulations, however, the effective surface tension has no lower bound and actually becomes negative. This removes the mechanism by which drops would pinch off. Future investigation into additional physics to describe tipstreaming involves the implementation of a nonlinear equation of state as described in [5]. Such a nonlinear equation of state cannot be implemented as a volume force as in this paper and will require a more sophisticated algorithm which includes a reconstruction of surface area. The ultimate aim is the simulation of droplet production up to its cessation, so that our scaling conditions on the total number of droplets, droplet size and capillary numbers could be verified.

\section{ACKNOWLEDGMENTS}

This research was sponsored by NSF-INT, NSF-DMS, NSF-CTS 0090381, and utilized the Illinois NCSA SGI Origin 2000. We are grateful to the Interdisciplinary Center for Applied Mathematics for the use of their Origin 2000. Acknowledgement is made to the

donors of The Petroleum Research Fund, administered by the ACS, for partial support of this research. 


\section{REFERENCES}

[1] M. Siegel. Influence of surfactant on rounded and pointed bubbles in 2D Stokes flow. SIAM J. Appl. Math, 59(6):1998-2027, 1999.

[2] Vittorio Cristini. Drop dynamics in viscous flow. PhD thesis, Yale University, 2000.

[3] C. D. Eggleton, T. M. Tsai, and K. J. Stebe. Tip streaming from a drop in the presence of surfactants. ICTAM 2000, QK2, 2000.

[4] R. A. De Bruijn. Tipstreaming of drops in simple shear flows. Chem. Eng. Scie., 48:277$284,1993$.

[5] Charles D. Eggleton, Yashodhara P. Pawar, and Kathleen J. Stebe. Insoluble surfactants on a drop in an extensional flow: a generalization of the stagnated surface limit to deforming interfaces. J. Fluid Mech., 385:79-99, 1999.

[6] Y. Wang, C. Maldarelli, and D. T. Papageorgiou. Increased mobility of a surfactant retarded bubble at high bulk concentrations. J. Fluid Mech., page in press, 2000.

[7] R. Scardovelli and S. Zaleski. Direct numerical simulation of free surface and interfacial flow. Ann. Rev. Fluid Mech., 31:567-604, 1999.

[8] D. Gueyffier, J. Li, A. Nadim, R. Scardovelli, and S. Zaleski. Volume-of-fluid interface tracking and smoothed surface stress methods for three-dimensional flows. J. Comp. Phys., 152:423-456, 1999.

[9] B. Lafaurie, C. Nardone, R. Scardovelli, S. Zaleski, and G. Zanetti. Modelling merging and fragmentation in multiphase flows with SURFER. J. Comp. Phys., 113:134-147, 1994.

[10] J. Li. Calcul d'Interface Affine par Morceaux (Piecewise Linear Interface Calculation). C. R. Acad. Sci. Paris, t.320 série IIb:391-396, 1995.

[11] J. Li, Y. Renardy, and M. Renardy. A numerical study of periodic disturbances on 
two-layer Couette flow. Phys. Fluids, 10:3056-3071, 1998.

[12] J. Li and Y. Renardy. Direct simulation of unsteady axisymmetric core-annular flow with high viscosity ratio. J. Fluid Mech., 391:123-149, 1999.

[13] J. Li, Y. Renardy, and M. Renardy. Numerical simulation of breakup of a viscous drop in simple shear flow through a volume-of-fluid method. Phys. Fluids, 12(2):269-282, 2000 .

[14] Yuriko Renardy and Jie Li. Parallelized simulations of two-fluid dispersions. SIAM News, in 'Applications on Advanced Architecture Computers', G. Astfalk ed.:December, 1, 2000.

[15] J. U. Brackbill, D. B. Kothe, and C. Zemach. A continuum method for modeling surface tension. J. Comp. Phys., 100:335-354, 1992.

[16] W. J. Rider and D. B. Kothe. Reconstructing volume tracking. J. Comp. Phys, 141:112$152,1998$.

[17] D.B. Kothe M.W. Williams and E.G. Puckett. Accuracy and convergence of continuum surface tension models. Fluid Dynamics at Interfaces (eds. W. Shyy and R. Narayanan), Cambridge Univ. Press, pages 294-305, 1998.

[18] H. A. Stone. Dynamics of drop deformation and breakup in viscous fluids. Ann. Rev. Fluid Mech., 26:65-102, 1994. 


\section{FIGURES}

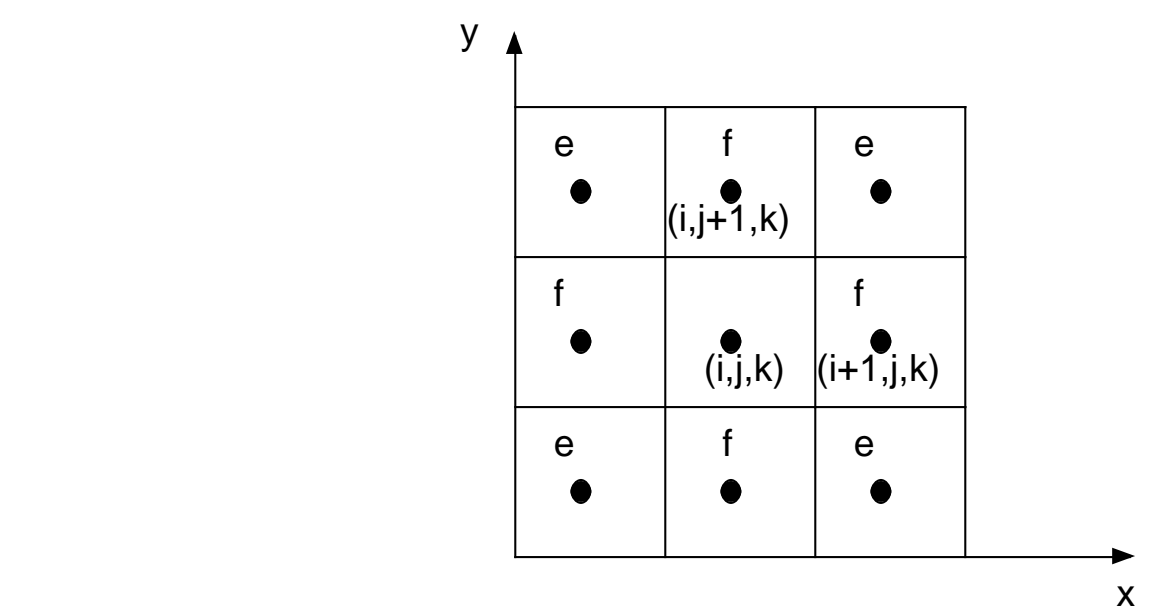

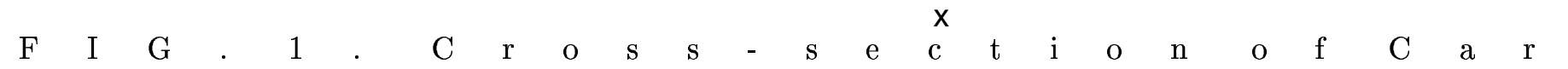

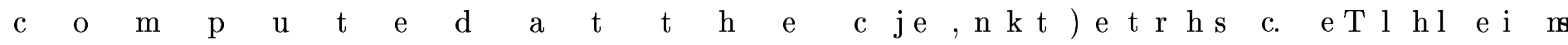
$\begin{array}{llllllllllllllllllllllllllll}K & 8 & \mathrm{k} & \mathrm{e} & \mathrm{r} & \mathrm{n} & \mathrm{e} & \mathrm{l} & , & \mathrm{a} & \mathrm{n} & \mathrm{d} & \mathrm{s} & \mathrm{u} & \mathrm{m} & \mathrm{m} & \mathrm{i} & \mathrm{n} & \mathrm{g} & \mathrm{c} & \mathrm{o} & \mathrm{l} & \mathrm{o} & \mathrm{r} & \mathrm{f}\end{array}$

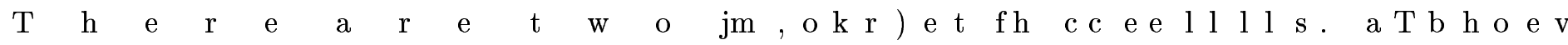

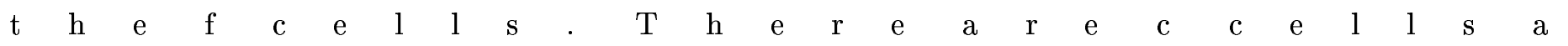




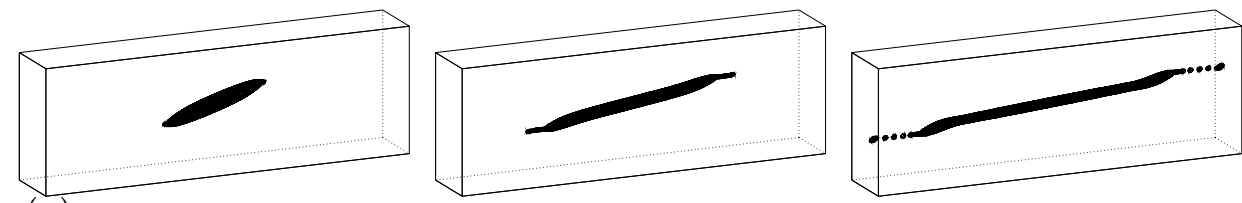

(a)

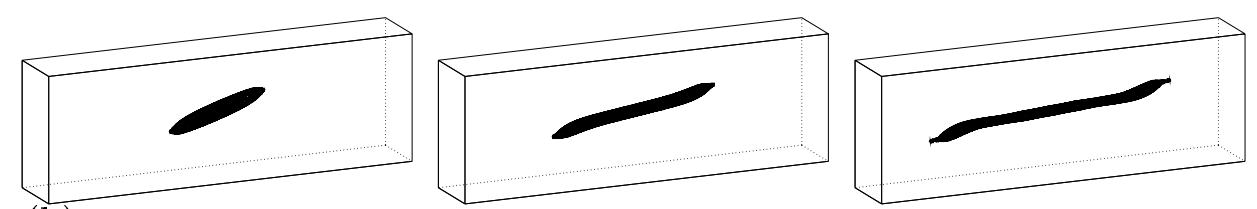

(b)

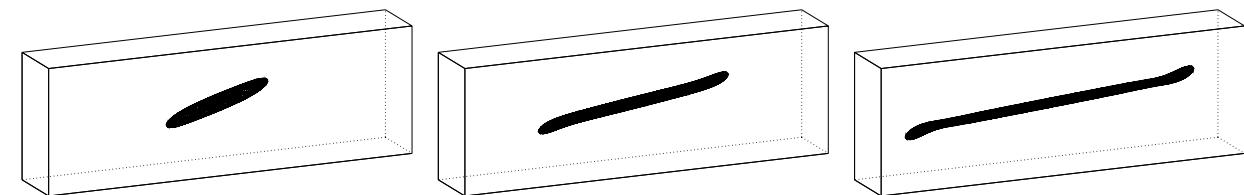

(c)

FIG. 2. $t=5,10,15 \mathrm{~s}$. (a) CSS with smoothing, (b) CSF with smoothing, (c) CSS without smoothing. $R e=1, C a=0.625, \lambda=0.05$. 


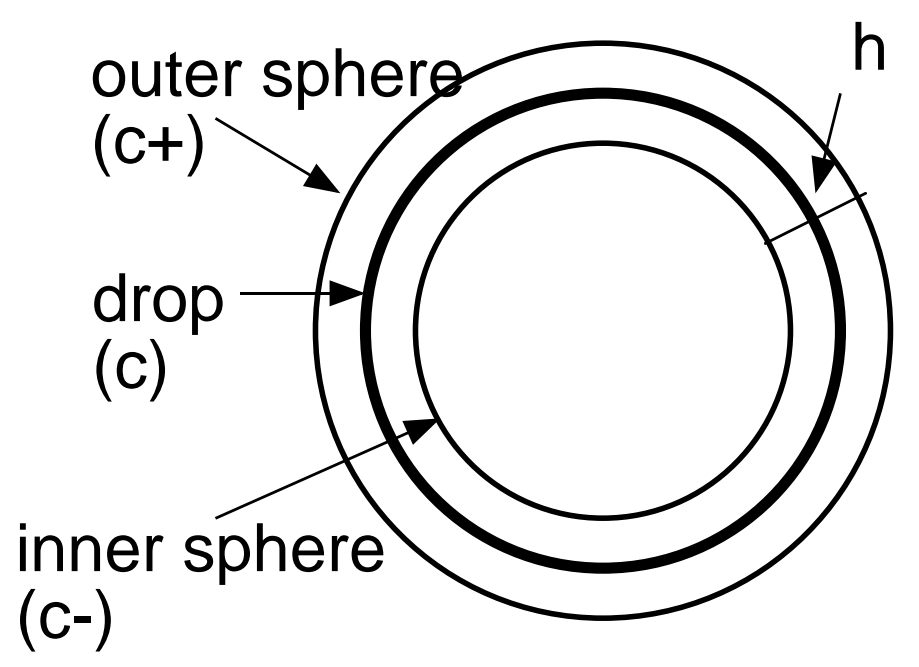

FIG. 3. Schematic of formulation for the inclusion of the linear equation of state for the surfactant concentration. The outer sphere is associated with the color function $c_{+}$, the inner sphere with $c_{-}$, the drop interface with $c$. 

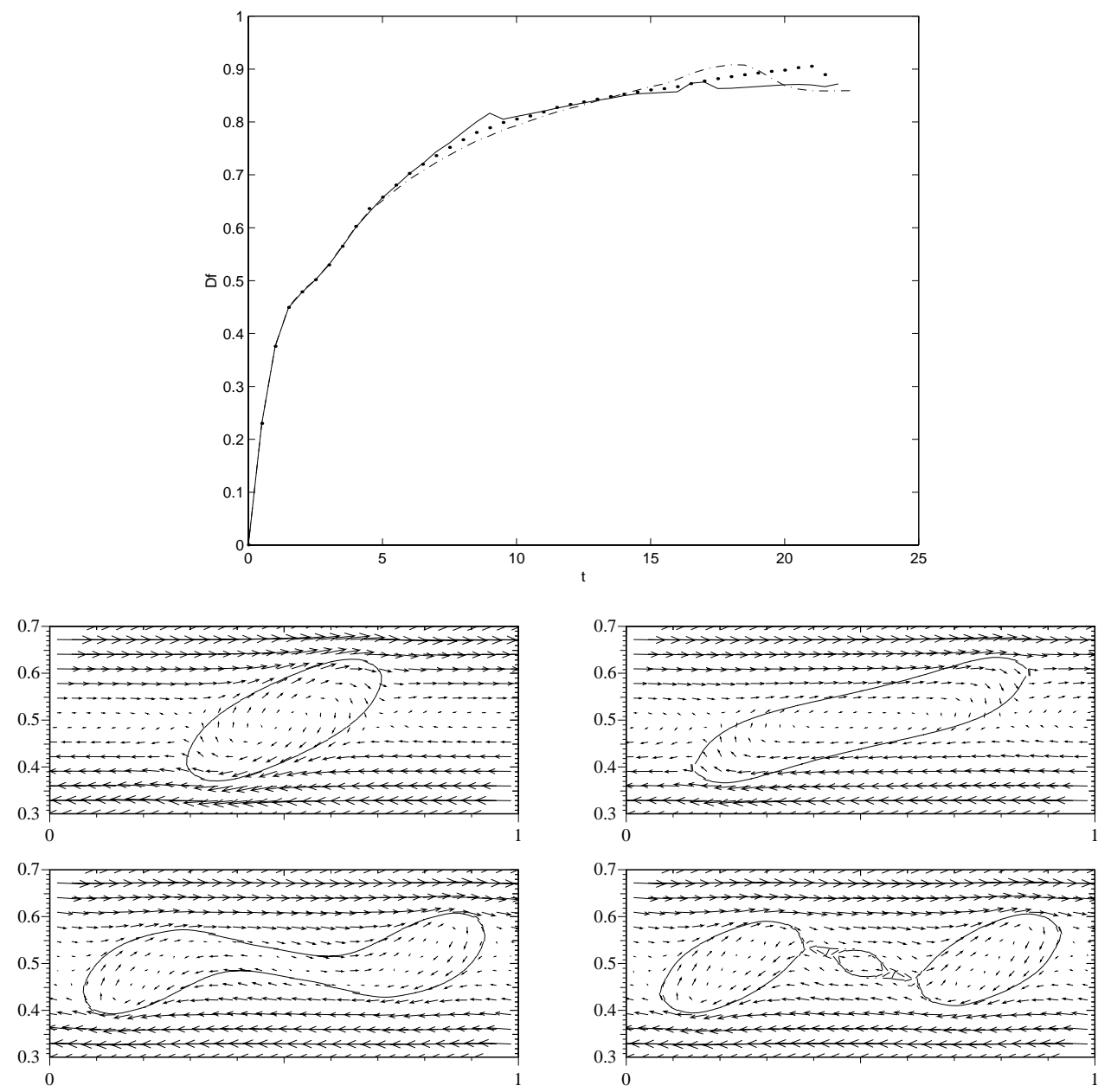

FIG. 4. Convergence study for surfactant layer thickness defined in Section IV. $R e=10, C a=0.16, r=0.2 .1 \times 0.5 \times 1$ box, $64 \times 32 \times 64$. Plot of deformation parameter Df vs $\mathrm{t}(\mathrm{s})$, for $h=$ mesh cell size 1/64 (-.), 1/2 mesh (.), 1/10 mesh (-), showing agreement for surfactant layer thickness of the order of the mesh cell size. Velocity fields are displayed at $\mathrm{t}=2.5$, $7.5,16.5,22 \mathrm{~s}$, for the $\mathrm{x}-\mathrm{z}$ cross-section through the center. The computational box is sized to induce the drop to interact with neighbors and develop the kinked neck. 

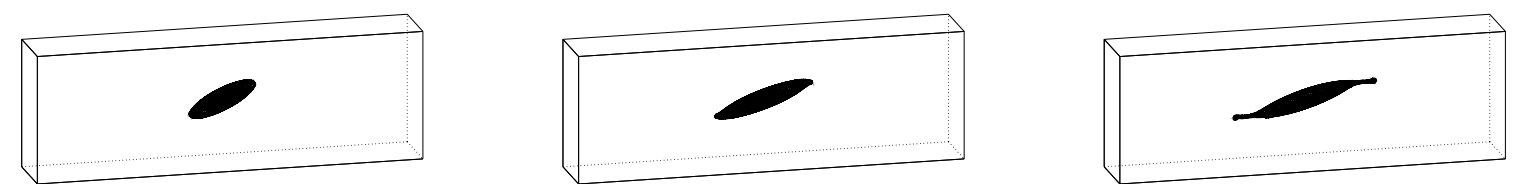

(a)
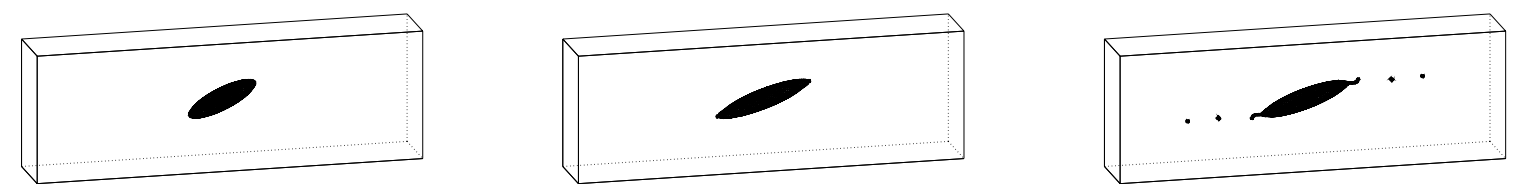

(b)

FIG. 5. Retention of surfactant on interfacial cells with the use of sufficiently thin initial surfactant layer. $R e=1, C a=0.2, r=0.3, t=4,8,12 \mathrm{~s} . h=(\mathrm{a}) \Delta x / 3$, (b) $\Delta x / 10$. Computational domain $3 \times 0.5 \times 1$, mesh $192 \times 32 \times 64$. 

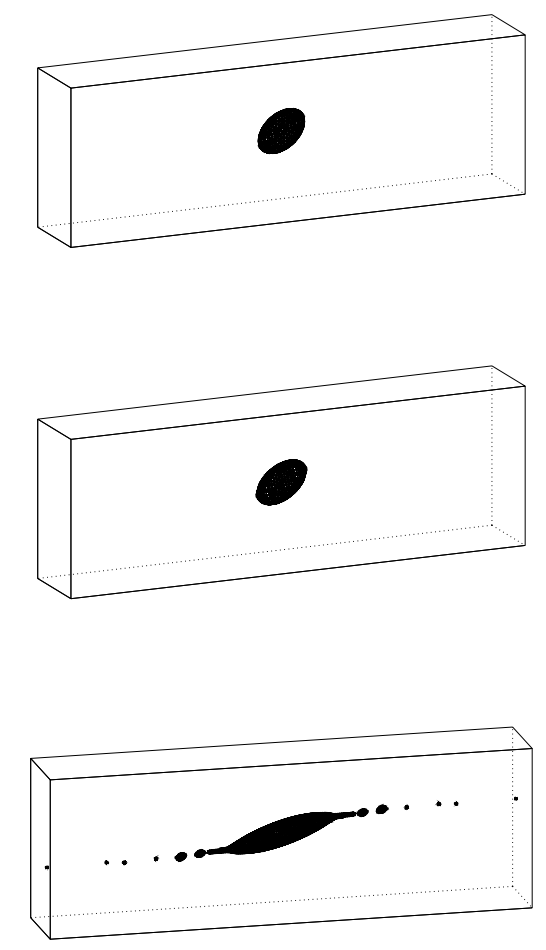

FIG. 6. $R e=1, C a=0.2, \lambda=0.05$. From top, $r=0$ (a steady solution is reached by $\mathrm{t}=2 \mathrm{~s}$ ), $r=0.1(\mathrm{t}=10 \mathrm{~s}), 0.3(\mathrm{t}=16 \mathrm{~s})$. 

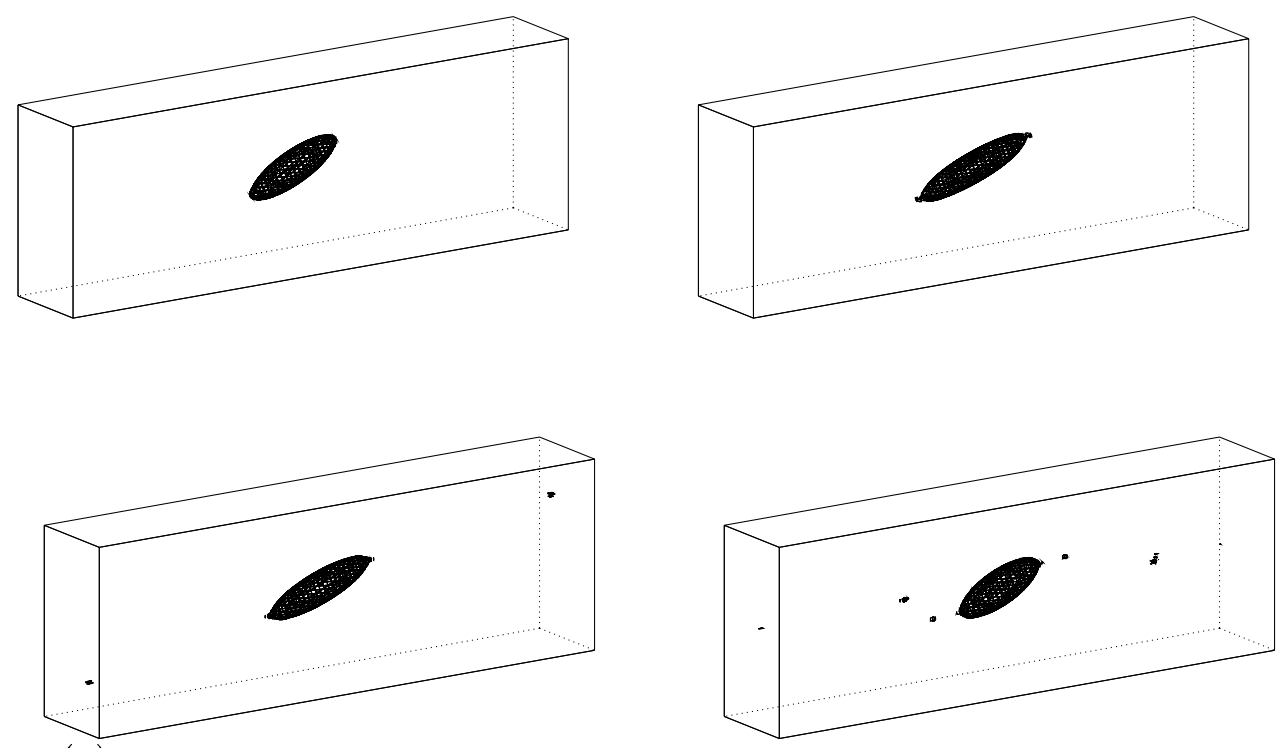

(a)
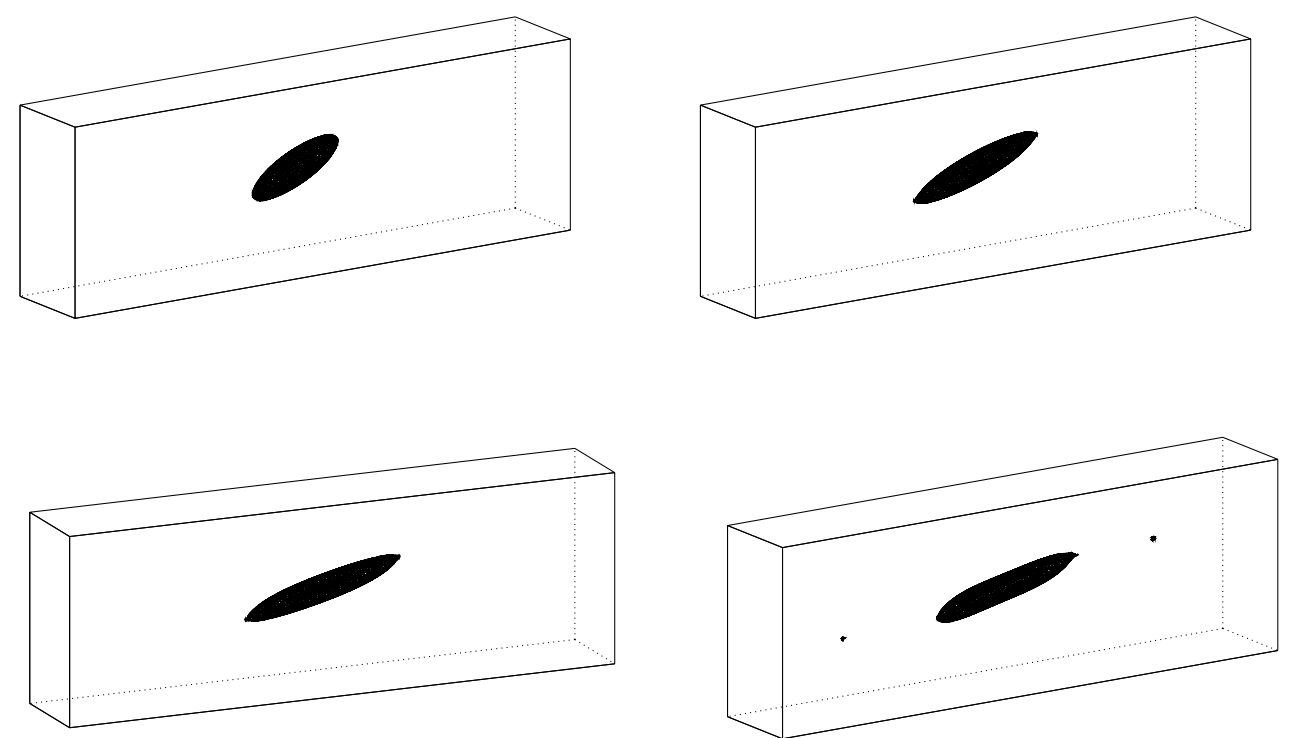

(b)

FIG. 7. Evolution of drop shape for $R e=1, C a=0.2, r=0.3, t=4,8,12,16 \mathrm{~s}$. (a) Mesh $128 \times 32 \times 64$. (b) Mesh $192 \times 48 \times 96$. 

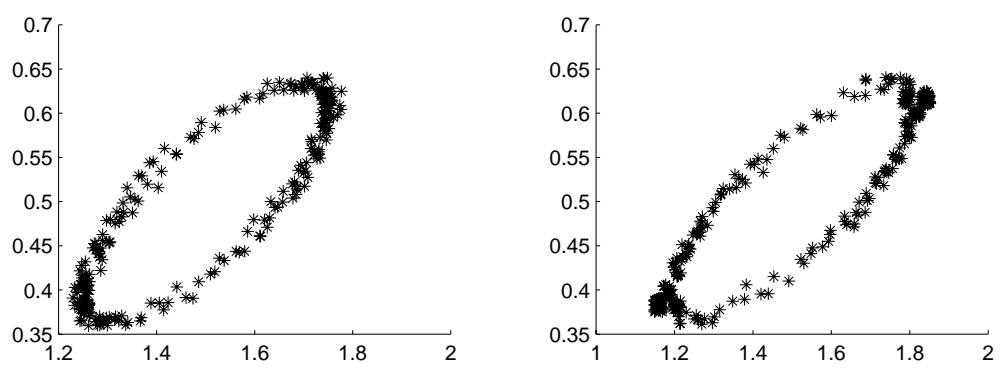

(a)
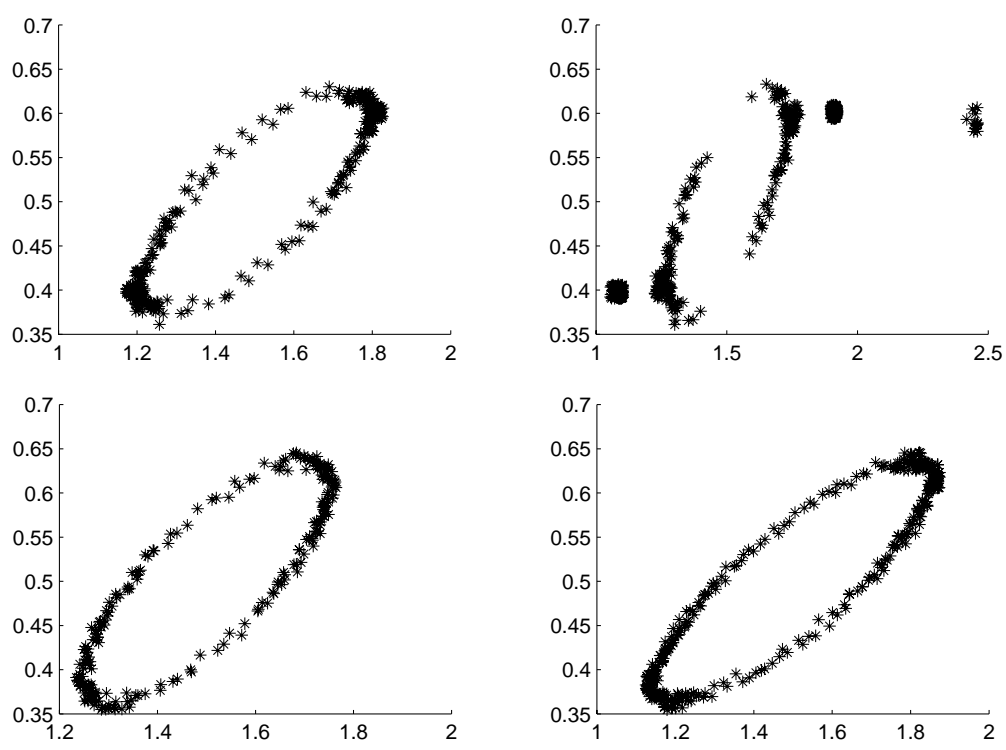

(b)
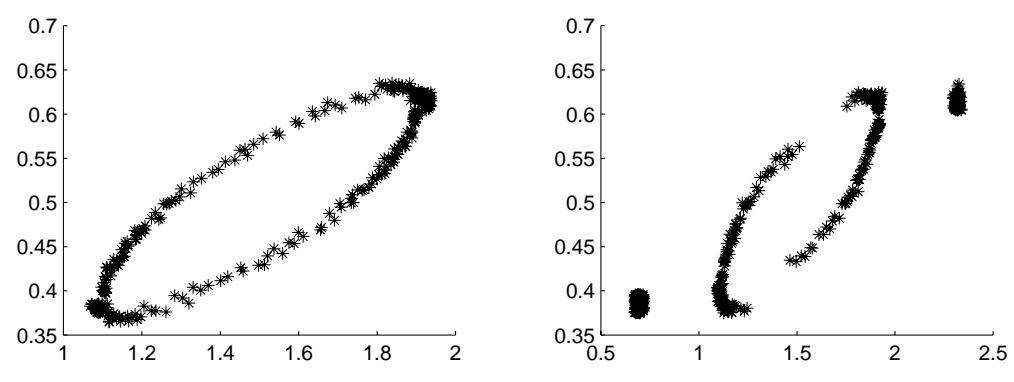

FIG. 8. Evolution of surfactant concentration for $R e=1, C a=0.2, r=0.3, t=4,8,12,16 \mathrm{~s}$.

(a) Mesh $128 \times 32 \times 64$. (b) Mesh $192 \times 48 \times 96$. 

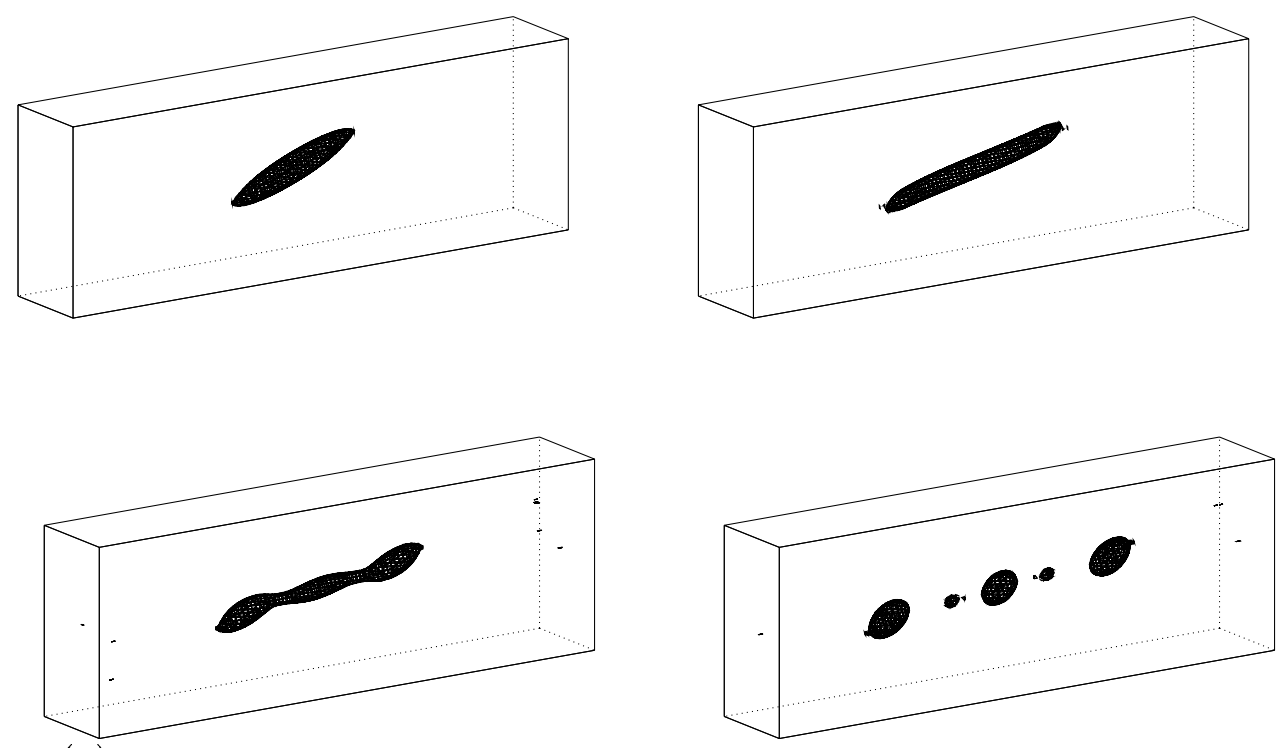

(a)
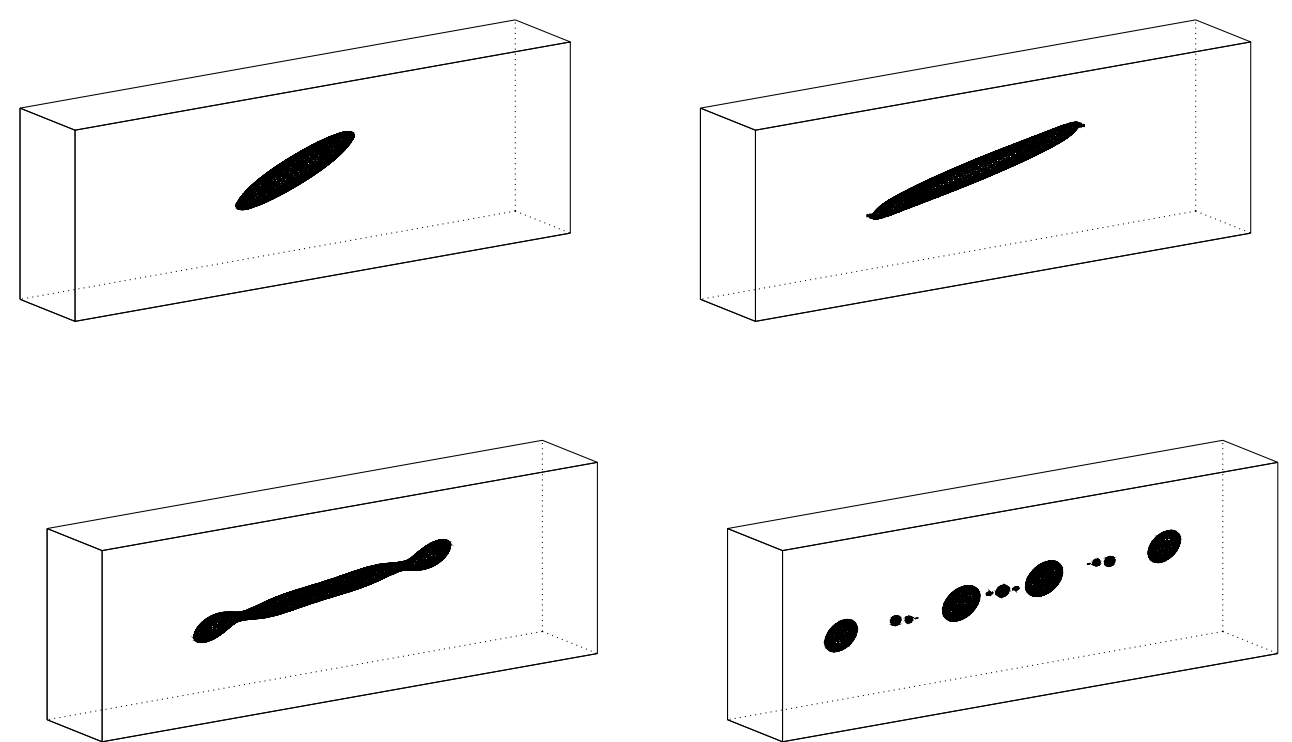

(b)

FIG. 9. Evolution of drop shape for $R e=1, C a=0.2, r=0.5, t=4,8,12,16 \mathrm{~s}$. (a) Mesh $128 \times 32 \times 64$. (b) Mesh $192 \times 48 \times 96$. 

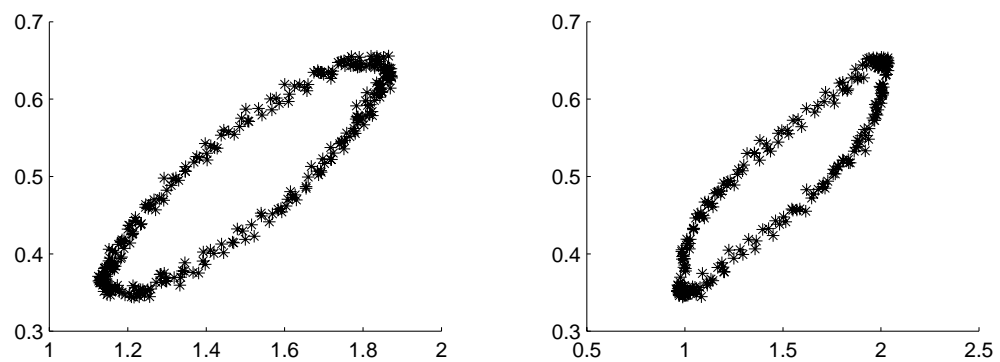

(a)
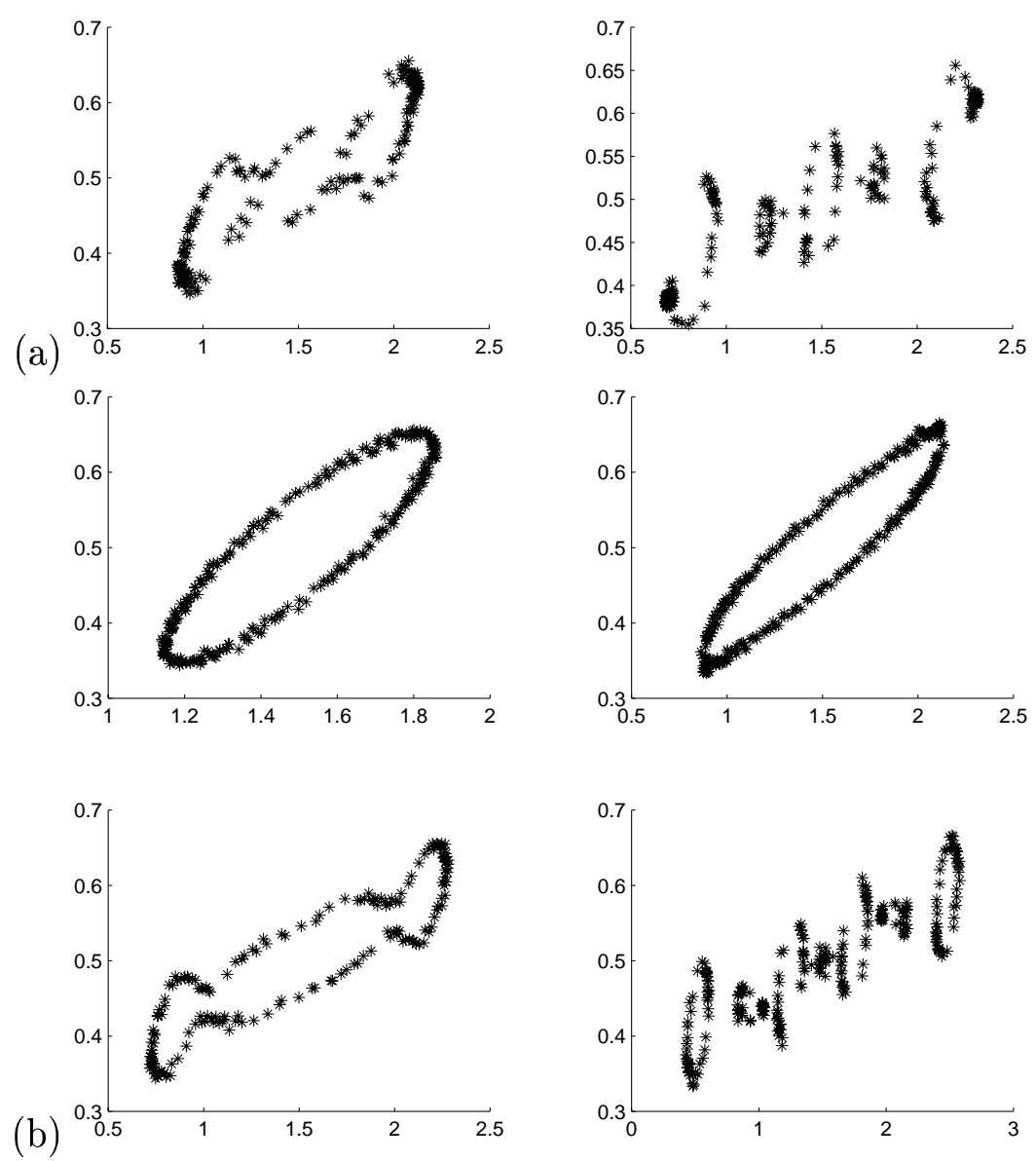

FIG. 10. Evolution of surfactant concentration for $R e=1, C a=0.2, r=0.5, t=4,8,12,16 \mathrm{~s}$.

(a) Mesh $128 \times 32 \times 64$. (b) Mesh $192 \times 48 \times 96$. 